ISSN 1995-5464 (Print); ISSN 2408-9524 (Online)

DOI: 10.16931/1995-5464.2019399-109

Границы операбельности при раке полжелуАочной железы: что лимитирует?

\author{
Субботин В.В. *, Душин И.Н., Забалуев Д.А., Камнев С.А., \\ Климов А.А., Малахова А.А., Осокин Я.А.
}

ГБУЗ “Московский клинический научный центр им. А.С. Логинова” ДЗМ; 111123, Москва, шоссе Энтузиастов, д. 86, Российская Федерация

\begin{abstract}
Предоперационная диагностика сопутствующих заболеваний необходима для выявления лимитирующих факторов в периоперационном периоде и правильной оценки возможностей пациента перенести предстоящее вмешательство. При наличии выраженных сопутствующих и фоновых заболеваний и, как следствие, высокой вероятности развития неблагоприятных событий необходимо разработать стратегию подготовки пациента к оперативному вмешательству и реализовать ее. Такая подготовка должна включать как медикаментозную, так и хирургическую коррекцию сопутствующих заболеваний для уменьшения возможных рисков. Таким образом, взвешенный, командный, мультидисциплинарный подход в периоперационном периоде позволяет значимо уменьшить риски и летальность у пациентов, оперируемых по поводу рака поджелудочной железы. Если мультидисциплинарной команде удается распознать и нейтрализовать лимитирующие факторы, то операбельность приближается к абсолютной, но если улучшения состояния пациента достичь не получается, то от операции следует воздержаться и применить другие методы лечения.
\end{abstract}

Ключевые слова: поджелудочная железа, рак, хирургическое вмешательство, предоперационная подготовка, факторы риска, сопутствующие заболевания, осложнения, летальность.

Ссылка для цитирования: Субботин В.В., Душин И.Н., Забалуев Д.А., Камнев С.А., Климов А.А., Малахова А.А., Осокин Я.А. Границы операбельности при раке поджелудочной железы: что лимитирует? Анналы хирургической гепатологии. 2019; 24 (3): 99-109. DOI: 10.16931/1995-5464.2019399-109.

Авторы заявляют об отсутствии конфликта интересов.

\title{
Operability boundaries in pancreatic cancer: what is the limit?
}

\author{
Subbotin V.V. *, Dushin I.N., Zabaluev D.A., Kamnev S.A., \\ Klimov A.A., Malakhova A.A., Osokin Ya.A. \\ Loginov Moscow Clinical Scientific Center of Moscow Department of Healthcare; Moscow, 86, Shosse \\ Entuziastov, Moscow, 111123, Russian Federation
}

\begin{abstract}
Preoperative diagnosis of comorbidities is essential for identifying the limiting factors inperioperative period and correct assessment of patient's ability to undergo surgery. Severe concomitant diseases followed by advanced risk of adverse events require development and implementation of the strategy of preoperative preparation of patient. These measuress hould include both medicamentous and surgical correction of concomitant diseases in order to reduce perioperative risks. Thus, multidisciplinary perioperative approach makes it possible to reduce significantly the risks and mortality in patients undergoing surgery for pancreatic cancer. Diagnosis and correction of limiting factors approach operability to absolute one. However, operation should be avoided or alternative approaches should be preferred if co-morbidities are significant and failed to be corrected.
\end{abstract}

Keywords: pancreas, cancer, surgical intervention, preoperative preparation, risk factors, concomitant diseases, complications, mortality.

For citation: Subbotin V.V., Dushin I.N., Zabaluev D.A., Kamnev S.A., Klimov A.A., Malakhova A.A., Osokin Ya.A. Operability boundaries in pancreatic cancer: what is the limit? Annaly khirurgicheskoy gepatologii = Annals of HPB surgery. $2019 ; 24$ (3): 99-109. (In Russian). DOI: 10.16931/1995-5464.2019399-109.

No conflict of interests to declare. 
Число и объем оперативных вмешательств растут год от года. По мнению экспертов, в 2010 г. было выполнено 321,5 млн хирургических процедур. Учитывая рост населения, этот показатель будет увеличиваться [1]. Немаловажную роль в этом будут играть достижения в области анестезиологии. В метаанализе 87 исследований, посвященных периоперационным осложнениям и охватывающих 21,4 млн пациентов, установлено, что смертность, связанная исключительно с анестезией, со временем уменьшилась. До 70-х гг. она составляла 357 на 1 млн (95\% ДИ $324-$ $394)$, в 70-80-х гг. - 52 на 1 млн (42-64), в 90-е и 2000-е гг. - 34 на 1 млн (29-39) $(p<0,00001)$. И это происходит несмотря на то, что физический статус пациентов, поступающих для оперативного лечения, из года в год ухудшается, а периоперационные риски развития осложнений растут [2].

Стоит признать, что в настоящее время периоперационные осложнения и летальность наиболее часто отмечают у пациентов высокого риска [3], к которым, как правило, относят людей с выраженными сопутствующими заболеваниями. В проведенных с 1952 по 2005 г. исследованиях было установлено, что периоперационная летальность в первую очередь связана с сопутствующими заболеваниями пациента, во вторую - со сложностью оперативного вмешательства и лишь в третью - с анестезиологическим пособием [4-7]. Исходя из этого можно предположить, что распознавание пациентов с высоким риском, предоперационная подготовка и специализированное интра- и послеоперационное ведение облегчают принятие хирургических решений и потенциально способны улучшить результаты лечения $[8,9]$.

К основным заболеваниям, ограничивающим хирургическое лечение пациентов с поражением поджелудочной железы (ПЖ), следует отнести сердечную недостаточность $(\mathrm{CH})$, ишемическую болезнь сердца (ИБС), нарушения ритма и проводимости миокарда, атеросклеротическое поражение сосудов, заболевания легких, хроническую болезнь почек (ХБП) и сахарный диабет (СД). СН является одним из самых распространенных заболеваний. Частота СН в развитых странах составляет $1-2 \%$, однако достигает $\geqslant 10 \%$ среди пациентов 70 лет и старше. Этот тезис подтверждают данные крупного регистра, включившего 160000 пациентов в возрасте 65 лет и старше, которым были выполнены медицинские вмешательства: $\mathrm{CH}$ наблюдалась у $18 \%$ больных $[10,11]$.

$\mathrm{CH}$ - хорошо известный предиктор развития периоперационных неблагоприятных кардиальных событий и важный фактор, включенный в большинство используемых шкал прогнозирования риска [12-14]. В первую очередь это связано с тем, что риск 30-дневной периоперацион- ной летальности у больных СН в 2-4 раза больше, чем у пациентов с изолированной ИБС, и превышает $8,5 \%$, даже после операций с низким кардиологическим риском [15]. Помимо этого, у пациентов с ИБС и признаками СН по сравнению с пациентами с ИБС без СН наблюдается увеличение риска периоперационной летальности на $63 \%$ и увеличение риска повторной госпитализации по любым причинам в течение 30 сут на 51\% [11]. Большое влияние на прогноз оказывает функциональное состояние левого желудочка. Было показано, что уменьшение фракции выброса левого желудочка $\leqslant 35 \%$ является сильным предиктором кардиальных осложнений после сосудистых хирургических вмешательств [16].

Другим, не менее важным заболеванием, приводящим к развитию периоперационных осложнений и летальности, является ИБС. Частота развития этих неблагоприятных событий при ИБС после некардиохирургических операций варьирует от 1 до 5\%. Немаловажным является тот факт, что на момент развития острого коронарного синдрома ИБС остается недиагностированной у $40 \%$ мужчин и $65 \%$ женщин. Несмотря на то что в настоящее время нарушения ритма в периоперационном периоде не являются самостоятельным фактором риска инфаркта миокарда или смерти, будь то единичные или множественные желудочковые экстрасистолы (ЖЭС) или даже "пробежки" желудочковой тахикардии, их наличие требует полноценного обследования пациента [17].

Частые гемодинамически незначимые ЖЭС (>60 в минуту) встречаются у $1-4 \%$ здоровых людей и протекают благоприятно [18]. Однако у кандидатов на хирургическое лечение рака ПЖ едва ли можно надеяться на такой вариант. Частота ЖЭС растет по мере развития сердечнососудистого заболевания и может достигать $90 \%$ при ИБС и дилятационной кардиомиопатии [19]. ЖЭС могут быть признаком не только заболеваний сердечно-сосудистой системы, но также метаболических и водно-электролитных нарушений, токсического действия препаратов [17]. Фибрилляция предсердий (ФП), самая частая тахиаритмия, сама по себе не является препятствием на пути к радикальному хирургическому лечению, но требует как непрерывной антиаритмической, так и адекватной антикоагулянтной терапии [17]. Наличие ФП у пациента обязывает обратить внимание на клапанный аппарат сердца и оценить степень развития у пациента ИБС.

Распространенность атеросклероза сонных артерий среди пациентов в возрасте старше 65 лет составляет $75 \%$ у мужчин и $62 \%$ у женщин. При этом двухлетний риск развития периоперационного инсульта при стенозе более $70 \%$ со- 
ставляет 26\%, что однозначно является неприемлемым [20]. Проблема мезентериального атеросклероза не менее остра, чем проблема атеросклероза сонных артерий. Доля атеросклероза мезентериальных артерий в структуре ишемии кишечника составляет порядка 5\%. Распространенность бессимптомного поражения мезентериальных артерий в популяции, к сожалению, изучена недостаточно [20]. Однако общепризнано, что наличие стеноза чревного ствола ухудшает прогноз и течение после резекции ПЖ, однако сам стеноз часто может клинически не проявляться ввиду наличия коллатералей с верхней брыжеечной артерией [21, 22].

Такие заболевания, как острые респираторные инфекции, хроническая обструктивная болезнь легких (ХОБЛ), бронхиальная астма, пневмосклероз, интерстициальные болезни легких и другие состояния, также оказывают значительное влияние на периоперационный риск. В большей степени это выражается в увеличении частоты послеоперационных легочных осложнений, которые увеличивают показатели заболеваемости и смертности. После серьезной внесердечной операции легочные осложнения встречаются так же часто, как и сердечные. На основании данных, полученных при проведении Национальной программы улучшения качества хирургии (NSQIP), было установлено, что послеоперационные легочные осложнения были у 6\% из 165196 пациентов, перенесших серьезные абдоминальные операции [23]. Кроме этого, установлено, что послеоперационные легочные осложнения достоверно связаны с повышением ранней (в течение 7 дней) послеоперационной летальности $-2,3 \%$ у пациентов с осложнениями и $0 \%$ у пациентов без них [24].

Особого внимания среди всех пациентов с заболеваниями легких требуют больные ХОБЛ и легочной гипертензией, поскольку именно эти состояния чаще остальных ассоциированы с заболеваниями сердечно-сосудистой системы, что в свою очередь еще больше увеличивает риск периоперационных осложнений и летальность. Распространенность ХОБЛ в Европе среди взрослого населения составляет 4-10\%. Легочная гипертензия (ЛАГ) - это гемодинамическое и патофизиологическое понятие, главным признаком которого является повышение среднего давления в легочной артерии $>25$ мм рт.ст. ЛАГ ассоциирована с увеличенной частотой послеоперационных осложнений, включая правожелудочковую недостаточность, ишемию миокарда и послеоперационную гипоксию. Ассоциированная частота сердечно-легочных периоперационных осложнений и летальность при этом заболевании составляют 38 и 7\% соответственно [10].

Хроническая болезнь почек в настоящее время является одним из самых распространен- ных неинфекционных заболеваний. В США ХБП 4-й и 5-й стадии выявляют у $0,6 \%$ населения, а ХБП любой стадии - у $33,2 \%$ населения в возрасте $\geq 60$ лет. Риск госпитализации, развития сердечно-сосудистых заболеваний и смерти растет при переходе на каждую следующую стадию ХБП [25]. При этом, например, ХБП 4-й или 5-й стадии приводит практически к десятикратному увеличению затрат на лечение пациента по любому поводу [26]. Известно, что ХБП является важным фактором риска неблагоприятного исхода крупных хирургических вмешательств. Пациенты с уровнем креатинина сыворотки $>160$ мкмоль/л после резекции ПЖ существенно чаще страдают послеоперационным острым повреждением почек и требуют проведения заместительной почечной терапии (ЗПТ). Такой уровень креатинина также является независимым фактором риска любых послеоперационных осложнений, в том числе дыхательной недостаточности и повторной интубации [27]. Пациенты с терминальной ХБП чаще подвержены развитию сепсиса и септического шока, кровотечения, требующего трансфузии. Они существенно больше времени проводят в стационаре после гемигепатэктомии и панкреатодуоденальной резекции $[28,29]$. Летальность среди пациентов с ХБП может быть почти в 10 раз больше, чем у прочих [29].

Сахарный диабет - это группа метаболических заболеваний, характеризующихся хронической гипергликемией, которая является результатом нарушения секреции инсулина, действия инсулина или обоих этих факторов [30]. В мире СД страдают 3-4\% взрослых людей, среди которых 95\% - это больные СД 2 типа. Каждый второй пациент с СД в течение жизни нуждается в оперативном лечении и анестезии. Главным фактором риска у пациентов с СД, подвергающихся хирургическому лечению, является поражение органов-мишеней, характерных для диабета: заболевания сердечно-сосудистой системы, почечная недостаточность, заболевания соединительной ткани (ограничение разгибания шеи, плохое заживление ран), неадекватный гранулоцитопоэз и нейропатии. Заболевание характеризуется множеством гормонально-индуцированных метаболических расстройств и диффузным повреждением микроциркуляторного русла. Инфекции у больных СД составляют порядка 2/3 послеоперационных осложнений и являются причиной послеоперационных смертельных исходов приблизительно в $20 \%$ наблюдений. Пациенты с СД чаще страдают инфекциями “чистых" ран, чем больные без диабета. В обзорном исследовании 23649 хирургических пациентов частота раневой инфекции “чистых" разрезов составила $10,7 \%$ у пациентов с СД и $1,8 \%$ у больных без СД [30, 31]. 
Кроме перечисленных сопутствующих заболеваний немаловажными факторами, лимитирующими оперативное лечение больных раком ПЖ, являются старческая астения, или хрупкость, и недоедание. Старческая астения - это возрастное прогрессирующее уменьшение множества физиологических резервов, приводящее к потере адаптационной способности и повышенной уязвимости к стрессовым воздействиям. Распространенность астении больше среди женщин, увеличивается с возрастом и отмечена у $40 \%$ пациентов в возрасте $\geqslant 80$ лет по сравнению с $10 \%$ у людей в возрасте 65-75 лет [32]. Для оценки старческой астении и связанных с ней рисков разработано несколько шкал (Шкала Эдмонтона Фрайля, Clinical Frailty Scale и др.), на основании которых было установлено, что при увеличении значения астении (хрупкости) возрастает число осложнений и летальность в разных областях хирургии [33-35].

Другим независимым предиктором высокой периоперационной летальности, продолжительности пребывания в стационаре и частоты повторных госпитализаций является недоедание. Основным критерием недоедания является индекс массы тела (ИМТ) $<18,5$ кг/м² [36]. Однако для пациентов пожилого возраста целесообразно использовать более высокую “отсечку" ИМТ как критерия недоедания. Проведенные исследования показывают, что риск смерти от всех причин начинает возрастать при ИМТ от $24 \mathrm{kг} / \mathrm{M}^{2}$ у пожилых и удваивается при ИМТ $<22$ кг $/ \mathrm{M}^{2}$ у мужчин и $<20 \mathrm{\kappa г} / \mathrm{M}^{2}$ у женщин [37]. Уровень сывороточного альбумина $<30$ г/л также является показателем недоедания, и, хотя его применение в качестве критерия подвергается критике, лучшего маркера для периоперационного нутритивного скрининга пока нет [38].

Таким образом, с учетом старения населения, коморбидность в хирургической практике будет встречаться все чаще, что требует периоперационной стратификации риска и принятия различных мер по уменьшению частоты осложнений и летальности у этой категории пациентов. Bce пациенты должны проходить стандартное предоперационное обследование, позволяющее выявить значимые сопутствующие заболевания и возможности их коррекции. Важнейшими вехами в комплексе диагностики являются правильный сбор анамнеза, оценка физического статуса и выявление имеющихся заболеваний [39].

При наличии сердечно-сосудистых заболеваний обязательно выполняют ЭКГ, ЭхоКГ, а при необходимости - нагрузочные пробы (тест с 6-минутной ходьбой, тредмил-тест, велоэргометрию, стресс-ЭхоКГ и перфузионную сцинтиграфию). В наиболее сложных клинических ситуациях необходимо выполнить коронарографию или мультиспиральную КТ-коронаро- графию для оценки проходимости венечных артерий. Пациентам с сердечной недостаточностью необходима трансторакальная ЭхоКГ и определение натрийуретических пептидов (BNP или NT proBNP), строго коррелирующих с уровнем пери- и послеоперационной заболеваемости и летальности [10, 40].

Для верификации болезней легких необходима оценка функции внешнего дыхания, диффузионной способности легких и, как следствие, выявление уровня дыхательной недостаточности. Кроме того, больным ожирением необходима полисомнография или STOP-BANG-опрос [39].

При наличии выраженного атеросклероза сосудов с признаками нарушения мозгового кровообращения или без них различные авторы рекомендуют выполнять УЗИ брахиоцефальных сосудов для уточнения степени сужения. По мнению исследователей, инсульт - наиболее частая причина смерти после операций на легких. Соответствующих данных по летальности в хирургии ПЖ не обнаружили, однако считаем, что заключение можно экстраполировать и сделать вывод, что УЗИ брахиоцефальных сосудов должно быть рутинным методом диагностики перед операцией на ПЖ [41, 42].

При подозрении или наличии ХБП рекомендуют выполнять биохимический анализ крови на содержание креатинина и мочевины с расчетом индекса клубочковой фильтрации [39].

Если у пациента есть сахарный диабет, то крайне важно установить степень его компенсации на основании уровня гликированного гемоглобина и текущих значений уровня глюкозы [43].

Учитывая изложенное, можно констатировать, что предоперационная диагностика позволяет выявить пациентов высокого риска и принять меры по устранению лимитирующих хирургическое лечение факторов. Данный тезис хорошо иллюстрирует модель "черного ящика" для индексов риска. Она гласит, что “при определении индекса риска частоту осложнений у пациентов с определенным фактором риска, перенесших операцию, принимают равной $(p)$. Если анестезиолог осведомлен о существенности данного фактора риска и может изменить периоперационную тактику анестезии, чтобы уменьшить этот риск $(p / 2)$, то этот фактор риска может потерять свою значимость. Однако если фактор риска игнорируется, у пациентов группы риска могут снова случаться осложнения" [44].

Тактику лечения больных СН III или IV функционального класса перед проведением общей анестезии согласовывают с кардиологом. В рекомендациях Европейского общества кардиологов по лечению острой и хронической $\mathrm{CH}$ представлены убедительные доказательства необходимости применения ингибиторов ангио- 
тезинпревращающего фермента в оптимально переносимых дозах, $\beta$-блокаторов и антагонистов альдостерона в качестве препаратов первого выбора [10]. При этом следует учитывать, что у пациентов с впервые диагностированной тяжелой систолической $\mathrm{CH}$ внесердечная операция должна быть отложена минимум на 3 мес, чтобы дать возможность новым лекарственным препаратам и лечебным вмешательствам благоприятно повлиять на систолическую функцию левого желудочка и ремоделирование [10]. Отсутствие положительной динамики следует рассматривать как ситуацию, требующую крайне взвешенного подхода к тактике ведения пациента.

При наличии ИБС предоперационная подготовка может включать медикаментозную коррекцию, хирургическую коррекцию путем открытой операции на сердце или эндоваскулярное вмешательство. В исследовании CASS летальность среди пациентов с ИБС, перенесших некардиохирургическую операцию, составила $2,4 \%$ без предоперационной реваскуляризации миокарда и $0,9 \%$ после предоперационной реваскуляризации методом коронарного шунтирования. Это существенное уменьшение, но ценой летальности во время кардиохирургической операции $-1,4 \%$. Мультивариантный анализ показал, что наибольшей пользой предоперационная реваскуляризация сопровождалась у пациентов с ранее перенесенным инфарктом, диабетом и средней и тяжелой ишемией по данным нагрузочных тестов [44]. Авторы оценили эффективность стентирования перед некардиохирургической операцией: эффект отмечали у пациентов, перенесших реваскуляризацию за 90 дней до операции, что в контексте основного онкологического заболевания достаточно большой срок. При выполнении операции в более ранние сроки после стентирования уменьшения частоты сердечно-сосудистых осложнений не наблюдали [44].

В другом исследовании оценили эффективность предупреждения сердечно-сосудистых осложнений у пациентов со стабильной ИБС без стволового поражения и с относительно сохранной фракцией выброса. Согласно опубликованным результатам, летальность через 2,7 года после рандомизации и частота послеоперационных кардиальных осложнений не различались.

Таким образом, вырисовывается характерная клиническая картина пациента, у которого реваскуляризация миокарда перед операцией окажет наибольшую пользу. Это пациент с тяжелой нестабильной ИБС, перенесший инфаркт миокарда, с диабетом. У него выявляют ишемические изменения на стресс-тестах и при коронарографии определяют стволовое поражение или его эквиваленты. Возможно значительное уменьшение фракции выброса на ЭхоКГ [44].
Bсе пациенты с симптомными аритмиями и нарушениями проводимости нуждаются в проведении полноценной терапии в предоперационном периоде, в том числе радиочастотной абляции и имплантации электрокардиостимуляторов или кардиовертер-дефибрилляторов по показаниям после консультации аритмологов. При этом наличие имплантированных устройств контроля ритма не является затруднением в периоперационном периоде. Тем не менее при подготовке пациентов с такими устройствами к операции следует иметь исчерпывающую документацию к устройству и сопровождающие документы из учреждения, осуществляющего ведение пациента.

Сами по себе нарушения ритма не сдвигают пределы операбельности больных раком ПЖ. Они являются важным тревожным сигналом при дооперационном обследовании пациента, поскольку нередко имеют под собой достаточно серьезные нарушения физиологии. Многие из них обратимы при адекватной предоперационной подготовке [45].

Каротидную эндартерэктомию (КЭАЭ) выполняют, если в будущем по основному заболеванию пациенту планируется оперативное вмешательство и у него высокий риск развития сосудистой церебральной катастрофы. Отечественные клинические рекомендации указывают на то, что симптомным пациентам (клиника транзиторных ишемических атак, острое нарушение мозгового кровообращения, головокружения с потерей сознания) со стенозом $>60 \%$ и бессимптомным пациентам со стенозом $>75 \%$ необходимо выполнить первым этапом КЭАЭ либо стентирование сонных артерий [41]. Кроме того, таким пациентам, по данным исследователей, показано применение статинов, поскольку они улучшают исход лечения пациентов с протоковой аденокарциномой ПЖ, не лечившихся до диагностики рака статинами, а также у пациентов с хроническим панкреатитом [46].

Стратегия лечения пациентов с заболеваниями легких перед операцией включает оптимизацию функции легких за счет проведения образовательных школ для пациентов, заблаговременное информирование пациента об отказе от курения (оптимально - не менее чем за 2 мес до операции), обучение принципам дыхательной гимнастики, тренировку дыхательной мускулатуры. Терапия $\beta$-агонистами и антихолинергическими средствами должна быть продолжена вплоть до дня операции у всех симптомных пациентов с ХОБЛ и признаками гиперреактивности бронхов. В отдельных наблюдениях может рассматриваться системная и (или) ингаляционная терапия стероидными гормонами. Пациенты с признаками сопутствующей правожелудочковой недостаточности должны получать соот- 
ветствующее лечение. При наличии признаков активной респираторной инфекции следует назначать антибиотикотерапию продолжительностью минимум 10 сут и при возможности хирургическое лечение должно быть отложено [47].

У пациентов с легочной гипертензией следует принимать во внимание потенциальную возможность развития острой правожелудочковой недостаточности и декомпенсации легочной гипертензии во время анестезии и самой операции, поэтому хирургические вмешательства следует выполнять только в случае крайней необходимости.

Обеспеченность населения заместительной почечной терапией в РФ неуклонно растет, опережая среднемировые показатели, и продолжительность жизни пациентов с ХБП увеличивается [48]. Почечная недостаточность - это лишь вершина айсберга, с которой можно успешно справиться. С одной стороны, проведение ЗПТ в послеоперационном периоде позволяет устранить все угрожающие жизни проблемы ХБП как таковой (гиперкалиемия и азотемия). С другой стороны, это не сможет уберечь пациентов от других сопутствующих заболеваний, например ИБС, которая является основной причиной смерти пациентов с ХБП [49]. Таким образом, хирургическое лечение рака ПЖ у пациентов с любой стадией ХБП, в том числе терминальной, несомненно, возможно и в некоторых клинических ситуациях успешно проводится [27-29]. Но неблагоприятный прогноз для таких пациентов как по основному заболеванию (с учетом, например, трудностей при проведении даже паллиативной химиотерапии) [50], так и по другим сопутствующим заболеваниям, в частности ИБС, требует взвешенного решения мультидисциплинарной комиссии с участием хирурга, онколога, нефролога, кардиолога и анестезиолога.

Наличие СД 1 и 2 типов само по себе не является противопоказанием к проведению операций на ПЖ. Однако большое значение имеет степень компенсации заболевания и, как следствие, уровень глюкозы крови. Во всех руководствах рекомендовано придерживаться следующих целевых концентраций глюкозы: до операции 7,8-10 ммоль/л, во время операции - 6,711,2 ммоль/л. Целевой уровень гликированного гемоглобина должен быть $<7 \%$. Если гликированный гемоглобин $>8,5 \%$, операцию следует отложить для дальнейшей подготовки [51], поскольку уровень гликированного гемоглобина является независимым предиктором фатального и нефатального инфаркта миокарда. Каждое повышение уровня гликированного гемоглобина на $1 \%$ больше нормы увеличивает риск внезапной коронарной смерти на $11 \%$ [52].

В отношении старческой астении и значимого недоедания можно рекомендовать оптимизацию питания, коррекцию метаболических нарушений и увеличение ежедневной функциональной активности [53], что, при возможности выполнения, может способствовать уменьшению частоты неблагоприятных исходов в лечении онкологического пациента. Однако при отсутствии положительной динамики от оперативного вмешательства лучше воздержаться.

На основании изложенного можно заключить, что возможность выполнения онкологической операции, ее целесообразность, а также целесообразность альтернативных методов лечения не должны оценивать исключительно хирурги или анестезиологи. Это требует проведения многопрофильного консилиума с активным привлечением смежных специалистов: кардиолога, пульмонолога, эндокринолога, сердечно-сосудистого хирурга, в том числе и специалиста по эндоваскулярным методам диагностики и лечения, нутрициолога и др.

Предоперационная диагностика сопутствующих заболеваний имеет первостепенную задачу выявления лимитирующих факторов в периоперационном периоде и правильной оценки возможностей организма перенести это вмешательство. При наличии выраженного морбидного фона и, как следствие, высокой вероятности развития неблагоприятных событий необходима разработка стратегии и реализация подготовки пациента к оперативному вмешательству. Такая подготовка должна включать как медикаментозную, так и хирургическую коррекцию сопутствующих заболеваний для уменьшения возможных рисков.

Другими словами, взвешенный, командный, мультидисциплинарный подход в периоперационном периоде дает возможность значимо уменьшить риски и вероятность летального исхода у пациентов, оперируемых по поводу рака ПЖ. Однако дуализм ситуации заключается в следующем: если мультидисциплинарной команде удается распознать и нейтрализовать лимитирующие факторы, то операбельность приближается к абсолютной, но если улучшения состояния пациента достичь не получается, несмотря на все предпринятые действия, то от операции следует воздержаться и предложить пациенту другие методы лечения.

\section{Участие авторов}

Субботин В.В. - концепция и дизайн статьи, написание текста, редактирование, утверждение окончательного варианта статьи.

Душин И.Н. - написание текста, редактирование. Забалуев Д.А. - написание текста, редактирование. Камнев С.А. - написание текста, редактирование. Климов А.А. - написание текста, редактирование. Малахова А.А. - написание текста, редактирование.

Осокин Я.А. - написание текста, редактирование. 


\section{Authors participation}

Subbotin V.V. - concept and design of the study, writing text, editing, approval of the final version of the article.

Dushin I.N. - writing text, editing.

Zabaluev D.A. - writing text, editing.

Kamnev S.A. - writing text, editing.

Klimov A.A. - writing text, editing.

Malakhova A.A. - writing text, editing.

Osokin J.A. - writing text, editing.

\section{Список литературы}

1. Rose J., Weiser T.G., Hider P., Wilson L., Gruen R.L., Bickler S.W. Estimated need for surgery worldwide based on prevalence of diseases: a modelling strategy for the WHO Global Health Estimate. Lancet Glob. Health. 2015; 3, Suppl 2: S13S20. http://doi.org/10.1016/S2214-109X(15)70087-2.

2. Bainbridge D., Martin J., Arango M., Cheng D. Evidence-based Peri-operative Clinical Outcomes Research (EPiCOR) Group. Perioperative and anaesthetic-related mortality in developed and developing countries: a systematic review and meta-analysis. Lancet. 2012; 380 (9847): 1075-1081. http://doi.org/10.1016/j.juro.2013.02.084.

3. Pearse R.M., Harrison D.A., James P., Watson D., Hinds C., Rhodes A., Grounds R.M., Bennett E.D. Identification and characterisation of the high-risk surgical population in the United Kingdom. Crit. Care. 2006; 10 (3): R81. http://doi.org/10.1186/cc4928.

4. Clifon B.S., Hotten W.I. Deaths associated with anaesthesia. $\mathrm{Br}$. J. Anaesth. 1963; 35: 250-259.

5. Marx G.F., Mateo C.V., Orkin L.R. Computer analysis of postanesthetic deaths. Anesthesiology. 1973; 39 (1): 54-58.

6. Cohen M.M., Duncan P.G., Pope W.D.B., Wolkenstein C. A survey of 112,000 anesthetics at one teaching hospital (1975-1983). Can. Anaesth. Soc. J. 1986; 33 (1): 22-31.

7. Monk T.G., Saini V., Weldon B.C., Sigl J.C. Anesthetic management and one-year mortality after noncardiac surgery. Anesth. Analg. 2005; 100 (1): 4-10. http://doi.org/10.1213/01.ANE.0000147519.82841.5E.

8. Moonesinghe S.R., Mythen M.G., Das P., Rowan K.M., Grocott M.P. Risk stratification tools for predicting morbidity and mortality in adult patients undergoing major surgery: qualitative systematic review. Anesthesiology. 2013; 119 (4): 959-981. http://doi.org/10.1097/ALN.0b013e3182a4e94d.

9. Bilimoria K.Y., Liu Y., Paruch J.L., Zhou L., Kmiecik T.E., Ko C.Y., Cohen M.E. Development and evaluation of the universal ACS NSQIP surgical risk calculator: a decision aid and informed consent tool for patients and surgeons. J. Am. Coll. Surg. 2013; 217 (5): 833-842.e1-3. http://doi.org/10.1016/j. jamcollsurg.2013.07.385.

10. Kristensen S.D., Knuuti J., Saraste A., Anker S., Bøtker H.E., De Hert S., Ford I., Gonzalez Juanatey J.R., Gorenek B., Heyndrickx G.R., Hoeft A., Huber K., Iung B., Kjeldsen K.P., Longrois D., Luescher T.F., Pierard L., Pocock S., Price S., Roffi M., Sirnes P.A., Uva M.S., Voudris V., Funck-Brentano C. Authors/Task Force Members. 2014 ESC/ESA guidelines on noncardiac surgery: cardiovascular assessment and management: The Joint Task Force on non-cardiac surgery: cardiovascular assessment and management of the European Society of Cardiology (ESC) and the European Society of Anaesthesiology (ESA). Eur. J. Anaesthesiol. 2014; 31 (10): 517-573. http://doi.org/10.1097/EJA.0000000000000150.

11. Hammill B.G., Curtis L.H., Bennett-Guerrero E., O'Connor C.M., Jollis J.G., Schulman K.A., Hernandez A.F.
Impact of heart failure on patients undergoing major noncardiac surgery. Anesthesiology. 2008; 108 (4): 559-567. http://doi.org/10.1097/ALN.0b013e31816725ef.

12. Goldman L., Caldera D.L., Nussbaum S.R., Southwick F.S., Krogstad D., Murray B., Burke D.S., O'Malley T.A., Goroll A.H., Caplan C.H., Nolan J., Carabello B., Slater E.E. Multifactorial index of cardiac risk in noncardiac surgical procedures. N. Engl. J. Med. 1977; 20; 297 (16): 845-850.

13. Detsky A.S., Abrams H.B., Forbath N., Scott J.G., Hilliard J.R. Cardiac assessment for patients undergoing noncardiac surgery. A multifactorial clinical risk index. Arch. Intern. Med. 1986; 146 (11): 2131-2134.

14. Lee T.H., Marcantonio E.R., Mangione C.M., Thomas E.J., Polanczyk C.A., Cook E.F., Sugarbaker D.J., Donaldson M.C., Poss R., Ho K.K., Ludwig L.E., Pedan A., Goldman L. Derivation and prospective validation of a simple index for prediction of cardiac risk of major noncardiac surgery. Circulation. 1999; 100 (10): 1043-1049.

15. van Diepen S., Bakal J.A., McAlister F.A., Ezekowitz J.A. Mortality and readmission of patients with heart failure, atrial fibrillation, or coronary artery disease undergoing noncardiac surgery: an analysis of 38047 patients. Circulation. 2011; 124 (3): 289-296. http://doi.org/10.1161/CIRCULATIONAHA.110.011130.

16. Kazmers A., Cerqueira M.D., Zierler R.E. Peri-operative and late outcome in patients with left ventricular ejection fraction of $35 \%$ or less who require major vascular surgery. J. Vasc. Surg. 1988; 8 (3): 307-315.

17. Fleisher L.A., Fleischmann K.E., Auerbach A.D., Barnason S.A., Beckman J.A., Bozkurt B., Davila-Roman V.G., GerhardHerman M.D., Holly T.A., Kane G.C., Marine J.E., Nelson M.T., Spencer C.C., Thompson A., Ting H.H., Uretsky B.F., Wijeysundera D.N. 2014 ACC/AHA Guideline on Perioperative Cardiovascular Evaluation and Management of Patients Undergoing Noncardiac Surgery. A Report of the American College of Cardiology/American Heart Association Task Force on Practice Guidelines. J. Am. Coll. Cardiol. 2014; 64 (22): e77-e137. http://doi.org/10.1016/j.jacc.2014.07.944.

18. Kennedy H.L., Whitlock J.A., Sprague M.K., Kennedy L.J., Buckingham T.A., Goldberg R.J. Long-term follow-up of asymptomatic healthy subjects with frequent and complex ventricular ectopy. N. Engl. J. Med. 1985; 312 (4): 193-197.

19. Perez-Silva A., Luis Merino J. Frequent ventricular extrasystoles: significance, prognosis and treatment. J. ESC Coun. Cardiol. Pract. 2011; 9 (17).

20. Tendera M., Aboyans V., Bartelink M.L., Baumgartner I., Clément D., Collet J.P., Cremonesi A., De Carlo M., Erbel R., Fowkes F.G., Heras M., Kownator S., Minar E., Ostergren J., Poldermans D., Riambau V., Roffi M., Röther J., Sievert H., van Sambeek M., Zeller T. ESC Committee for Practice Guidelines. ESC Guidelines on the diagnosis and treatment of peripheral artery diseases: Document covering atherosclerotic disease of extracranial carotid and vertebral, mesenteric, renal, upper and lower extremity arteries: the Task Force on the Diagnosis and Treatment of Peripheral Artery Diseases of the European Society of Cardiology (ESC). Eur. Heart. J. 2011; 32 (22): 2851-2906. http://doi.org/10.1093/eurheartj/ehr211. Epub 2011 Aug 26.

21. Giovanardi F., Lai Q., Garofalo M., Arroyo Murillo G.A., Choppin de Janvry E., Hassan R., Larghi Laureiro Z., Consolo A., Melandro F., Berloco P.B. Collaterals management during pancreatoduodenectomy in patients with celiac axis stenosis: A systematic review of the literature. Pancreatology. 2018; pii: S1424-3903(18)30087-5. http://doi.org/10.1016/j.pan.2018.05.003. 
22. Nara S., Sakamoto Y., Shimada K., Sano T., Kosuge T., Takahashi Y., Onaya H., Yamamoto J. Arterial reconstruction during pancreatoduodenectomy in patients with celiac axis stenosis -utility of doppler ultrasonography. World J. Surg. 2005; 29 (7): 885-889. http://doi.org/10.1007/s00268-005-7878-x.

23. Yang C.K., Teng A., Lee D.Y., Rose K. Pulmonary complications after major abdominal surgery: National Surgical Quality Improvement Program analysis. J. Surg. Res. 2015; 198 (2): 441-449. http://doi.org/10.1016/j.jss.2015.03.028.

24. Fernandez-Bustamante A., Frendl G., Sprung J., Kor D.J., Subramaniam B., Martinez Ruiz R., Lee J.W., Henderson W.G., Moss A., Mehdiratta N., Colwell M.M., Bartels K., Kolodzie K., Giquel J., Vidal Melo M.F. Postoperative pulmonary complications, early mortality, and hospital stay following noncardiothoracic surgery: a multicenter study by the perioperative research network investigators. JAMA Surg. 2017; 152 (2): 157-166. http://doi.org/10.1001/jamasurg.2016.4065.

25. Benjamin E.J., Blaha M.J., Chiuve S.E., Cushman M., Das S.R., Deo R., de Ferranti S.D., Floyd J., Fornage M., Gillespie C., Isasi C.R., Jiménez M.C., Jordan L.C., Judd S.E., Lackland D., Lichtman J.H., Lisabeth L., Liu S., Longenecker C.T., Mackey R.H., Matsushita K., Mozaffarian D., Mussolino M.E., Nasir K., Neumar R.W., Palaniappan L., Pandey D.K., Thiagarajan R.R., Reeves M.J., Ritchey M., Rodriguez C.J., Roth G.A., Rosamond W.D., Sasson C., Towfighi A., Tsao C.W., Turner M.B., Virani S.S., Voeks J.H., Willey J.Z., Wilkins J.T., Wu J.H., Alger H.M., Wong S.S., Muntner P. American Heart Association Statistics Committee and Stroke Statistics Subcommittee. Heart disease and stroke statistics-2017 update: a report from the American Heart Association. Circulation. 2017; 135 (10): e146-e603.

http://doi.org/10.1161/CIR.0000000000000485.

26. Golestaneh L., Alvarez P.J., Reaven N.L., Funk S.E., McGaughey K.J., Romero A., Brenner M.S., Onuigbo M. Allcause costs increase exponentially with increased chronic kidney disease stage. Am. J. Manag. Care. 2017; 23 (10 Suppl): S163-S172.

27. Squires M.H. 3rd, Mehta V.V., Fisher S.B., Lad N.L., Kooby D.A., Sarmiento J.M., Cardona K., Russell M.C., Staley C.A. 3rd, Maithel S.K. Effect of preoperative renal insufficiency on postoperative outcomes after pancreatic resection: a single institution experience of 1,061 consecutive patients. J. Am. Coll. Surg. 2014; 218 (1): 92-101. http://doi.org/0.1016/j.jamcollsurg.2013.09.012.

28. Barbas A.S., Speicher P.J., Clary B.M. Hepatic and pancreatic resection in patients with end-stage renal disease: a propensity analysis. HPB (Oxford). 2014; 16 (11): 1016-1022. http://doi.org/10.1111/hpb.12281.

29. Norman K.S., Domingo S.R., Wong L.L. Pancreatic resections in renal failure patients: is it worth the risk? HPB Surg. 2014; 2014: 938251. http://doi.org/10.1155/2014/938251.

30. Дедов И.И., Шестакова М.В., Галстян Г.Р. Распространенность сахарного диабета 2-го типа у взрослого населения России (исследование NATION). Сахарный диабет. 2016; 2: 104-112.

31. Cruse P.J., Foord R. A 5-year prospective study of 23649 surgical wounds. Arch. Surg. 1973; 107 (2): 206-210.

32.Collard R.M., Boter H., Schoevers R.A., Oude Voshaar R.C. Prevalence of frailty in community-dwelling older persons: a systematic review. J. Am. Geriatr. Soc. 2012; 60 (8): 1487-1492. http://doi.org/10.1111/j.1532-5415.2012.04054.X.

33. Robinson T.N., Wu D.S., Sauaia A., Dunn C.L., StevensLapsley J.E., Moss M., Stiegmann G.V., Gajdos C., Cleveland J.C. Jr., Inouye S.K. Slower walking speed forecasts increased postoperative morbidity and one year mortality across surgical specialties. Ann. Surg. 2013; 258 (4): 582-588; discussion 588-590. http://doi.org/10.1097/SLA.0b013e3182a4e96c.

34. Rockwood K., Song X., MacKnight C., Bergman H., Hogan D.B., McDowell I., Mitnitski A. A global clinical measure of fitness and frailty in elderly people. CMAJ. 2005; 173 (5): 489-495.

35. Hewitt J., Carter B., McCarthy K., Pearce L., Law J., Wilson F.V., Tay H.S., McCormack C., Stechman M.J., Moug S.J., Myint P.K. Frailty predicts mortality in all emergency surgical admissions regardless of age. An observational study. Age Ageing. 2019. pii: afy217. http://doi.org/10.1093/ageing/afy217.

36. Weimann A., Braga M., Carli F., Higashiguchi T., Hübner M., Klek S., Laviano A., Ljungqvist O., Lobo D.N., Martindale R., Waitzberg D.L., Bischoff S.C., Singer P. ESPEN guideline: clinical nutrition in surgery. Clin. Nutr. 2017; 36 (3): 623-650. http://doi.org/10.1016/j.clnu.2017.02.013.

37. Sergi G., Perissinotto E., Pisent C., Buja A., Maggi S., Coin A., Grigoletto F., Enzi G., ILSA Working Group. An adequate threshold for body mass index to detect underweight condition in elderly persons: the Italian Longitudinal Study on Aging (ILSA). J. Gerontol. A. Biol. Sci. Med. Sci. 2005; 60 (7): 866-871.

38. Nelson C.L., Elkassabany N.M., Kamath A.F., Liu J. Low albumin levels, more than morbid obesity, are associated with complications after TKA. Clin. Orthop. Relat. Res. 2015; 473 (10): 3163-3172. http://doi.org/10.1007/s11999-015-4333-7.

39. De Hert S., Staender S., Fritsch G., Hinkelbein J., Afshari A., Bettelli G., Bock M., Chew M.S., Coburn M., De Robertis E., Drinhaus H., Feldheiser A., Geldner G., Lahner D., Macas A., Neuhaus C., Rauch S., Santos-Ampuero M.A., Solca M., Tanha N., Traskaite V., Wagner G., Wappler F. Pre-operative evaluation of adults undergoing elective noncardiac surgery. Eur. J. Anaesthesiol. 2018; 35 (6): 407-465. http://doi.org/10.1097/EJA.0000000000000817.

40. Bose S., Talmor D. Who is a high-risk surgical patient? Curr. Opin. Crit. Care. 2018; 24 (6): 547-553. http://doi.org/10.1097/MCC.0000000000000556.

41. Национальные рекомендации по ведению пациентов с заболеваниями брахиоцефальных артерий. Ангиология и сосудистая хирургия. 2013; 19: 73.

42. Shoji F., Takeo S., Yamazaki K., Miura N., Katsura M., Oku Y., Shimokawa M. Impact of preoperative ultrasonography screening for carotid artery stenosis in lung cancer patients. Ann. Thorac. Surg. 2018; 106 (4): 1047-1054. http://doi.org/10.1016/j.athoracsur.2018.04.072.

43. Клинические рекомендации "Периоперационное ведение пациентов с сопутствующим сахарным диабетом”. Общероссийская общественная организация "Федерация анестезиологов и реаниматологов”, 2017. 55 с.

44. Miller R.D., Cohen N.H., Eriksson L.I., Fleisher L.A., WienerKronish J.P., Young W.L. Eighth edition. 2015. 1071 p.

45. Клинические рекомендации “Желудочковые аритмии". Общероссийская общественная организация “Федерация анестезиологов и реаниматологов”, 2018. 47 с.

46. Jeon C.Y., Pandol S.J., Wu B., Cook-Wiens G., Gottlieb R.A., Merz C.N., Goodman M.T. The association of statin use after cancer diagnosis with survival in pancreatic cancer patients: A SEER-Medicare analysis. PLoS One. 2015; 10 (4): e0121783. http://doi.org/10.1371/journal.pone.0121783.

47. Edrich T., Sadovnikoff N. Anesthesia for patients with severe chronic obstructive pulmonary disease. Curr. Opin. Anaesthesiol. 2010;23:18-24.http://doi.org/10.1097/ACO.0b013e328331ea5b.

48. Томилина Н.А., Андрусев А.М., Перегудова Н.Г., Шинкарев М.Б. Заместительная терапия терминальной хронической почечной недостаточности в Российской Федерации в 2010-2015 гг. Нефрология и диализ. 2017; 19 (4): 93 с. Приложение. 
49. Thompson S., James M., Wiebe N., Hemmelgarn B., Manns B., Klarenbach S., Tonelli M.; Alberta Kidney Disease Network. Cause of death in patients with reduced kidney function. J. Am. Soc. Nephrol. 2015; 26 (10): 2504-2511. http://doi.org/10.1681/ASN.2014070714.

50. Funakoshi T., Horimatsu T., Nakamura M., Shiroshita K., Suyama K., Mukoyama M., Mizukami T., Sakurada T., Baba E., Tsuruya K., Nozaki A., Yahata K., Ozaki Y., Ubara Y., Yasui H., Yoshimoto A., Fukuma S., Kondo N., Matsubara T., Matsubara K., Fukuhara S., Yanagita M., Muto M. Chemotherapy in cancer patients undergoing haemodialysis: a nationwide study in Japan. ESMO Open. 2018; 3 (2): e000301. http://doi.org/10.1136/esmoopen-2017-000301.

51. Standard of Medical Care in Diabetes. American Diabetes Association. Clin. Diabetes. 2017; 35 (1): 5-26. http://doi.org/10.2337/cd16-0067.

52. Abadal A.C., Flores J.S. The significance of hyperglycemia in acute coronary syndrome. Rev. Esp. Cardiol. 2008; 61 (5): 447-450. http://doi.org/10.1016/S1885-5857(08)60156-5.

53. Arrieta H., Astrugue C., Regueme S., Durrieu J., Maillard A., Rieger A., Terrebonne E., Laurent C., Maget B., Servent V., Lavau-Denès S., Dauba J., Fonck M., Thiébaut R., BourdelMarchasson I. Effects of a physical activity programme to prevent physical performance decline in onco-geriatric patients: a randomized multicentre trial. J. Cachexia Sarcopenia Muscle. 2019; 10 (2): 287-297. http://doi.org/10.1002/jcsm.12382.

\section{References}

1. Rose J., Weiser T.G., Hider P., Wilson L., Gruen R.L., Bickler S.W. Estimated need for surgery worldwide based on prevalence of diseases: a modelling strategy for the WHO Global Health Estimate. Lancet Glob. Health. 2015; 3, Suppl 2: S13S20. http://doi.org/10.1016/S2214-109X(15)70087-2.

2. Bainbridge D., Martin J., Arango M., Cheng D. Evidence-based Peri-operative Clinical Outcomes Research (EPiCOR) Group. Perioperative and anaesthetic-related mortality in developed and developing countries: a systematic review and meta-analysis. Lancet. 2012; 380 (9847): 1075-1081. http://doi.org/10.1016/j.juro.2013.02.084.

3. Pearse R.M., Harrison D.A., James P., Watson D., Hinds C., Rhodes A., Grounds R.M., Bennett E.D. Identification and characterisation of the high-risk surgical population in the United Kingdom. Crit. Care. 2006; 10 (3): R81. http://doi.org/10.1186/cc4928.

4. Clifon B.S., Hotten W.I. Deaths associated with anaesthesia. Br. J. Anaesth. 1963; 35: 250-259.

5. Marx G.F., Mateo C.V., Orkin L.R. Computer analysis of postanesthetic deaths. Anesthesiology. 1973; 39 (1): 54-58.

6. Cohen M.M., Duncan P.G., Pope W.D.B., Wolkenstein C. A survey of 112,000 anesthetics at one teaching hospital (1975-1983). Can. Anaesth. Soc. J. 1986; 33 (1): 22-31.

7. Monk T.G., Saini V., Weldon B.C., Sigl J.C. Anesthetic management and one-year mortality after noncardiac surgery. Anesth. Analg. 2005; 100 (1): 4-10. http://doi.org/10.1213/01.ANE.0000147519.82841.5E.

8. Moonesinghe S.R., Mythen M.G., Das P., Rowan K.M., Grocott M.P. Risk stratification tools for predicting morbidity and mortality in adult patients undergoing major surgery: qualitative systematic review. Anesthesiology. 2013; 119 (4): 959-981. http://doi.org/10.1097/ALN.0b013e3182a4e94d.

9. Bilimoria K.Y., Liu Y., Paruch J.L., Zhou L., Kmiecik T.E., Ko C.Y., Cohen M.E. Development and evaluation of the universal ACS NSQIP surgical risk calculator: a decision aid and informed consent tool for patients and surgeons. J. Am. Coll.
Surg. 2013; 217 (5): 833-842.e1-3. http://doi.org/10.1016/j. jamcollsurg.2013.07.385.

10. Kristensen S.D., Knuuti J., Saraste A., Anker S., Bøtker H.E., De Hert S., Ford I., Gonzalez Juanatey J.R., Gorenek B., Heyndrickx G.R., Hoeft A., Huber K., Iung B., Kjeldsen K.P., Longrois D., Luescher T.F., Pierard L., Pocock S., Price S., Roffi M., Sirnes P.A., Uva M.S., Voudris V., Funck-Brentano C. Authors/Task Force Members. 2014 ESC/ESA guidelines on noncardiac surgery: cardiovascular assessment and management: The Joint Task Force on non-cardiac surgery: cardiovascular assessment and management of the European Society of Cardiology (ESC) and the European Society of Anaesthesiology (ESA). Eur. J. Anaesthesiol. 2014; 31 (10): 517-573. http://doi.org/10.1097/EJA.0000000000000150.

11. Hammill B.G., Curtis L.H., Bennett-Guerrero E., O'Connor C.M., Jollis J.G., Schulman K.A., Hernandez A.F. Impact of heart failure on patients undergoing major noncardiac surgery. Anesthesiology. 2008; 108 (4): 559-567. http://doi.org/10.1097/ALN.0b013e31816725ef.

12. Goldman L., Caldera D.L., Nussbaum S.R., Southwick F.S., Krogstad D., Murray B., Burke D.S., O'Malley T.A., Goroll A.H., Caplan C.H., Nolan J., Carabello B., Slater E.E. Multifactorial index of cardiac risk in noncardiac surgical procedures. N. Engl. J. Med. 1977; 20; 297 (16): 845-850.

13. Detsky A.S., Abrams H.B., Forbath N., Scott J.G., Hilliard J.R. Cardiac assessment for patients undergoing noncardiac surgery. A multifactorial clinical risk index. Arch. Intern. Med. 1986; 146 (11): 2131-2134.

14. Lee T.H., Marcantonio E.R., Mangione C.M., Thomas E.J., Polanczyk C.A., Cook E.F., Sugarbaker D.J., Donaldson M.C., Poss R., Ho K.K., Ludwig L.E., Pedan A., Goldman L. Derivation and prospective validation of a simple index for prediction of cardiac risk of major noncardiac surgery. Circulation. 1999; 100 (10): 1043-1049.

15. van Diepen S., Bakal J.A., McAlister F.A., Ezekowitz J.A. Mortality and readmission of patients with heart failure, atrial fibrillation, or coronary artery disease undergoing noncardiac surgery: an analysis of 38047 patients. Circulation. 2011; 124 (3): 289-296. http://doi.org/10.1161/CIRCULATIONAHA.110.011130.

16. Kazmers A., Cerqueira M.D., Zierler R.E. Peri-operative and late outcome in patients with left ventricular ejection fraction of $35 \%$ or less who require major vascular surgery. J. Vasc. Surg. 1988; 8 (3): 307-315.

17. Fleisher L.A., Fleischmann K.E., Auerbach A.D., Barnason S.A., Beckman J.A., Bozkurt B., Davila-Roman V.G., GerhardHerman M.D., Holly T.A., Kane G.C., Marine J.E., Nelson M.T., Spencer C.C., Thompson A., Ting H.H., Uretsky B.F., Wijeysundera D.N. 2014 ACC/AHA Guideline on Perioperative Cardiovascular Evaluation and Management of Patients Undergoing Noncardiac Surgery. A Report of the American College of Cardiology/American Heart Association Task Force on Practice Guidelines. J. Am. Coll. Cardiol. 2014; 64 (22): e77-e137. http://doi.org/10.1016/j.jacc.2014.07.944.

18. Kennedy H.L., Whitlock J.A., Sprague M.K., Kennedy L.J., Buckingham T.A., Goldberg R.J. Long-term follow-up of asymptomatic healthy subjects with frequent and complex ventricular ectopy. N. Engl. J. Med. 1985; 312 (4): 193-197.

19. Perez-Silva A., Luis Merino J. Frequent ventricular extrasystoles: significance, prognosis and treatment. J. ESC Coun. Cardiol. Pract. 2011; 9 (17).

20. Tendera M., Aboyans V., Bartelink M.L., Baumgartner I., Clément D., Collet J.P., Cremonesi A., De Carlo M., Erbel R., Fowkes F.G., Heras M., Kownator S., Minar E., Ostergren J., Poldermans D., Riambau V., Roffi M., Röther J., Sievert H., 
van Sambeek M., Zeller T. ESC Committee for Practice Guidelines. ESC Guidelines on the diagnosis and treatment of peripheral artery diseases: Document covering atherosclerotic disease of extracranial carotid and vertebral, mesenteric, renal, upper and lower extremity arteries: the Task Force on the Diagnosis and Treatment of Peripheral Artery Diseases of the European Society of Cardiology (ESC). Eur. Heart. J. 2011; 32 (22): 2851-2906. http://doi.org/10.1093/eurheartj/ehr211. Epub 2011 Aug 26.

21. Giovanardi F., Lai Q., Garofalo M., Arroyo Murillo G.A., Choppin de Janvry E., Hassan R., Larghi Laureiro Z., Consolo A., Melandro F., Berloco P.B. Collaterals management during pancreatoduodenectomy in patients with celiac axis stenosis: A systematic review of the literature. Pancreatology. 2018; pii: S1424-3903(18)30087-5. http://doi.org/10.1016/j.pan.2018.05.003.

22. Nara S., Sakamoto Y., Shimada K., Sano T., Kosuge T., Takahashi Y., Onaya H., Yamamoto J. Arterial reconstruction during pancreatoduodenectomy in patients with celiac axis stenosis - utility of doppler ultrasonography. World J. Surg. 2005; 29 (7): 885-889. http://doi.org/10.1007/s00268-005-7878-x.

23. Yang C.K., Teng A., Lee D.Y., Rose K. Pulmonary complications after major abdominal surgery: National Surgical Quality Improvement Program analysis. J. Surg. Res. 2015; 198 (2): 441-449. http://doi.org/10.1016/j.jss.2015.03.028.

24. Fernandez-Bustamante A., Frendl G., Sprung J., Kor D.J., Subramaniam B., Martinez Ruiz R., Lee J.W., Henderson W.G., Moss A., Mehdiratta N., Colwell M.M., Bartels K., Kolodzie K., Giquel J., Vidal Melo M.F. Postoperative pulmonary complications, early mortality, and hospital stay following noncardiothoracic surgery: a multicenter study by the perioperative research network investigators. JAMA Surg. 2017; 152 (2): 157-166. http://doi.org/10.1001/jamasurg.2016.4065.

25. Benjamin E.J., Blaha M.J., Chiuve S.E., Cushman M., Das S.R., Deo R., de Ferranti S.D., Floyd J., Fornage M., Gillespie C., Isasi C.R., Jiménez M.C., Jordan L.C., Judd S.E., Lackland D., Lichtman J.H., Lisabeth L., Liu S., Longenecker C.T., Mackey R.H., Matsushita K., Mozaffarian D., Mussolino M.E., Nasir K., Neumar R.W., Palaniappan L., Pandey D.K., Thiagarajan R.R., Reeves M.J., Ritchey M., Rodriguez C.J., Roth G.A., Rosamond W.D., Sasson C., Towfighi A., Tsao C.W., Turner M.B., Virani S.S., Voeks J.H., Willey J.Z., Wilkins J.T., Wu J.H., Alger H.M., Wong S.S., Muntner P. American Heart Association Statistics Committee and Stroke Statistics Subcommittee. Heart disease and stroke statistics-2017 update: a report from the American Heart Association. Circulation. 2017; 135 (10): e146-e603.

http://doi.org/10.1161/CIR.0000000000000485.

26. Golestaneh L., Alvarez P.J., Reaven N.L., Funk S.E., McGaughey K.J., Romero A., Brenner M.S., Onuigbo M. Allcause costs increase exponentially with increased chronic kidney disease stage. Am. J. Manag. Care. 2017; 23 (10 Suppl): S163-S172.

27. Squires M.H. 3rd, Mehta V.V., Fisher S.B., Lad N.L., Kooby D.A., Sarmiento J.M., Cardona K., Russell M.C., Staley C.A. 3rd, Maithel S.K. Effect of preoperative renal insufficiency on postoperative outcomes after pancreatic resection: a single institution experience of 1,061 consecutive patients. J. Am. Coll. Surg. 2014; 218 (1): 92-101. http://doi.org/0.1016/j.jamcollsurg.2013.09.012.

28. Barbas A.S., Speicher P.J., Clary B.M. Hepatic and pancreatic resection in patients with end-stage renal disease: a propensity analysis. HPB (Oxford). 2014; 16 (11): 1016-1022. http://doi.org/10.1111/hpb.12281.
29. Norman K.S., Domingo S.R., Wong L.L. Pancreatic resections in renal failure patients: is it worth the risk? HPB Surg. 2014; 2014: 938251. http://doi.org/10.1155/2014/938251.

30. Dedov I.I., Shestakova M.V., Galstyan G.R. Prevalence of diabetes mellitus type 2 in adult population of Russia (NATION study). Saharnyiy diabet. 2016; 2: 104-112. (In Russian)

31. Cruse P.J., Foord R. A 5-year prospective study of 23649 surgical wounds. Arch. Surg. 1973; 107 (2): 206-210.

32.Collard R.M., Boter H., Schoevers R.A., Oude Voshaar R.C. Prevalence of frailty in community-dwelling older persons: a systematic review. J. Am. Geriatr. Soc. 2012; 60 (8): 1487-1492. http://doi.org/10.1111/j.1532-5415.2012.04054.x.

33. Robinson T.N., Wu D.S., Sauaia A., Dunn C.L., StevensLapsley J.E., Moss M., Stiegmann G.V., Gajdos C., Cleveland J.C. Jr., Inouye S.K. Slower walking speed forecasts increased postoperative morbidity and one year mortality across surgical specialties. Ann. Surg. 2013; 258 (4): 582-588; discussion 588-590. http://doi.org/10.1097/SLA.0b013e3182a4e96c.

34. Rockwood K., Song X., MacKnight C., Bergman H., Hogan D.B., McDowell I., Mitnitski A. A global clinical measure of fitness and frailty in elderly people. CMAJ. 2005; 173 (5): 489-495.

35. Hewitt J., Carter B., McCarthy K., Pearce L., Law J., Wilson F.V., Tay H.S., McCormack C., Stechman M.J., Moug S.J., Myint P.K. Frailty predicts mortality in all emergency surgical admissions regardless of age. An observational study. Age Ageing. 2019. pii: afy217. http://doi.org/10.1093/ageing/afy217.

36. Weimann A., Braga M., Carli F., Higashiguchi T., Hübner M., Klek S., Laviano A., Ljungqvist O., Lobo D.N., Martindale R., Waitzberg D.L., Bischoff S.C., Singer P. ESPEN guideline: clinical nutrition in surgery. Clin. Nutr. 2017; 36 (3): 623-650. http://doi.org/10.1016/j.clnu.2017.02.013.

37. Sergi G., Perissinotto E., Pisent C., Buja A., Maggi S., Coin A., Grigoletto F., Enzi G., ILSA Working Group. An adequate threshold for body mass index to detect underweight condition in elderly persons: the Italian Longitudinal Study on Aging (ILSA). J. Gerontol. A. Biol. Sci. Med. Sci. 2005; 60 (7): 866-871.

38. Nelson C.L., Elkassabany N.M., Kamath A.F., Liu J. Low albumin levels, more than morbid obesity, are associated with complications after TKA. Clin. Orthop. Relat. Res. 2015; 473 (10): 3163-3172. http://doi.org/10.1007/s11999-015-4333-7.

39. De Hert S., Staender S., Fritsch G., Hinkelbein J., Afshari A., Bettelli G., Bock M., Chew M.S., Coburn M., De Robertis E., Drinhaus H., Feldheiser A., Geldner G., Lahner D., Macas A., Neuhaus C., Rauch S., Santos-Ampuero M.A., Solca M., Tanha N., Traskaite V., Wagner G., Wappler F. Pre-operative evaluation of adults undergoing elective noncardiac surgery. Eur. J. Anaesthesiol. 2018; 35 (6): 407-465. http://doi.org/10.1097/EJA.0000000000000817.

40. Bose S., Talmor D. Who is a high-risk surgical patient? Curr. Opin. Crit. Care. 2018; 24 (6): 547-553. http://doi.org/10.1097/MCC.0000000000000556.

41. Nacional'nye rekomendacii po vedeniyu pacientov s zabolevaniyami brahiocefal'nyh arterij [National guidelines for the management of patients with brachiocephalic artery disease]. Angiologiya $i$ sosudistaya khirurgiya. 2013; 19: 73. (In Russian)

42. Shoji F., Takeo S., Yamazaki K., Miura N., Katsura M., Oku Y., Shimokawa M. Impact of preoperative ultrasonography screening for carotid artery stenosis in lung cancer patients. Ann. Thorac. Surg. 2018; 106 (4): 1047-1054. http://doi.org/10.1016/j.athoracsur.2018.04.072.

43. Klinicheskie rekomendacii "Perioperacionnoe vedenie pacientov s soputstvuyushchim saharnym diabetom”. Obshcherossijskaya obshchestvennaya organizaciya "Federaciya anesteziologov i reanimatologov" [Clinical guidelines. Perioperative management 
of patients with diabetes mellitus. Federation of anesthesiologists and reanimatologists]. 2017. 55 p. (In Russian)

44. Miller R.D., Cohen N.H., Eriksson L.I., Fleisher L.A., WienerKronish J.P., Young W.L. Eighth edition. 2015. 1071 p.

45. Klinicheskie rekomendacii "Zheludochkovye aritmii". Obshcherossijskaya obshchestvennaya organizaciya "Federaciya anesteziologov $i$ reanimatologov" [Clinical guidelines "Ventricular arrhythmias". Federation of anesthesiologists and reanimatologists]. 2018. 47 p. (In Russian)

46. Jeon C.Y., Pandol S.J., Wu B., Cook-Wiens G., Gottlieb R.A., Merz C.N., Goodman M.T. The association of statin use after cancer diagnosis with survival in pancreatic cancer patients: A SEER-Medicare analysis. PLoS One. 2015; 10 (4): e0121783. http://doi.org/10.1371/journal.pone.0121783.

47. Edrich T., Sadovnikoff N. Anesthesia for patients with severe chronic obstructive pulmonary disease. Curr. Opin. Anaesthesiol. 2010;23:18-24.http://doi.org/10.1097/ACO.0b013e328331ea5b.

48. Tomilina N.A., Andrusev A.M., Peregudova N.G., Shinkarev M.B. Renal replacement the rapy for terminal chronic renal failure in the Russian Federation in 2010-2015. Nefrologiya i dializ. 2017; 19 (4): 93 p. Supplement. (In Russian)

49. Thompson S., James M., Wiebe N., Hemmelgarn B., Manns B., Klarenbach S., Tonelli M.; Alberta Kidney Disease Network. Cause of death in patients with reduced kidney function.
J. Am. Soc. Nephrol. 2015; 26 (10): 2504-2511.

http://doi.org/10.1681/ASN.2014070714.

50. Funakoshi T., Horimatsu T., Nakamura M., Shiroshita K., Suyama K., Mukoyama M., Mizukami T., Sakurada T., Baba E., Tsuruya K., Nozaki A., Yahata K., Ozaki Y., Ubara Y., Yasui H., Yoshimoto A., Fukuma S., Kondo N., Matsubara T., Matsubara K., Fukuhara S., Yanagita M., Muto M. Chemotherapy in cancer patients undergoing haemodialysis: a nationwide study in Japan. ESMO Open. 2018; 3 (2): e000301. http://doi.org/10.1136/esmoopen-2017-000301.

51. Standard of Medical Care in Diabetes. American Diabetes Association. Clin. Diabetes. 2017; 35 (1): 5-26. http://doi.org/10.2337/cd16-0067.

52. Abadal A.C., Flores J.S. The significance of hyperglycemia in acute coronary syndrome. Rev. Esp. Cardiol. 2008; 61 (5): 447-450. http://doi.org/10.1016/S1885-5857(08)60156-5.

53. Arrieta H., Astrugue C., Regueme S., Durrieu J., Maillard A., Rieger A., Terrebonne E., Laurent C., Maget B., Servent V., Lavau-Denès S., Dauba J., Fonck M., Thiébaut R., BourdelMarchasson I. Effects of a physical activity programme to prevent physical performance decline in onco-geriatric patients: a randomized multicentre trial. J. Cachexia Sarcopenia Muscle. 2019; 10 (2): 287-297. http://doi.org/10.1002/jcsm.12382.

\section{Сведения об авторах [Authors info]}

Субботин Валерий Вячеславович - доктор мед. наук, заведующий центром анестезиологии-реаниматологии ГБУз МКНЦ им. А.С. Логинова ДЗМ. http://orcid.org/0000-0002-0921-7199. E-mail: v.subbotin@mknc.ru

Душин Иван Николаевич - врач анестезиолог-реаниматолог отделения анестезиологии-реанимации №1 ГБУЗ МКНЦ им. А.С. Логинова ДЗМ. http://orcid.org/0000-0002-5857-2998. E-mail: ivan-dushin@mail.ru

Забалуев Денис Алексеевич - врач анестезиолог-реаниматолог отделения анестезиологии-реанимации №1 ГБУ3 МКНЦ им. А.С. Логинова ДЗМ. http://orcid.org/0000-0003-4887-1166. E-mail: d.zabaluev@mknc.ru

Камнев Сергей Анатольевич - врач анестезиолог-реаниматолог отделения анестезиологии-реанимации №1 ГБУ3 МКНЦ им. А.С. Логинова ДЗМ. https://orcid.org/0000-0002-4230-4796. E-mail: korium1605@gmail.com

Климов Андрей Андреевич - врач анестезиолог-реаниматолог отделения анестезиологии-реанимации №1 ГБУ3 МКНЦ им. А.С. Логинова ДЗМ. http://orcid.org/0000-0001-7633-6852. E-mail: an.an.klimov@gmail.com

Малахова Анна Анатольевна - врач анестезиолог-реаниматолог отделения анестезиологии-реанимации №1 ГБУ3 МКНЦ им. А.С. Логинова ДЗМ. http://orcid.org/0000-0002-2918-5091. E-mail: an.an.malahova@yandex.ru

Осокин Яков Александрович - клинический ординатор отделения анестезиологии-реанимации №1 ГБУЗ МКНЦ им. А.С. Логинова ДЗМ. http://orcid.org/0000-0003-4013-4970. E-mail: jamnstuff@gmail.com

Для корреспонденции *: Субботин Валерий Вячеславович - 111123, Москва, шоссе Энтузиастов, д. 86, Российская Федерация. Тел.: +7-916-612-25-04. E-mail: v.subbotin@mknc.ru

Valeriy V. Subbotin - Doct. of Sci. (Med.), Head of the ICU Department, Loginov Moscow Clinical Scientific Center of Moscow Department of Healthcare. http://orcid.org/0000-0002-0921-7199. E-mail: v.subbotin@mknc.ru

Ivan N. Dushin - ICU-physician of the ICU Department №1, Loginov Moscow Clinical Scientific Center of Moscow Department of Healthcare. http://orcid.org/0000-0002-5857-2998. E-mail: ivan-dushin@mail.ru

Denis A. Zabaluev - ICU-physician of the ICU Department №1, Loginov Moscow Clinical Scientific Center of Moscow Department of Healthcare. http://orcid.org/0000-0003-4887-1166. E-mail: d.zabaluev@mknc.ru

Sergey A. Kamnev - ICU-physician of the ICU Department №1, Loginov Moscow Clinical Scientific Center of Moscow Department of Healthcare. http://orcid.org/0000-0002-4230-4796. E-mail: korium1605@gmail.com

Andrey A. Klimov - ICU-physician of the ICU Department №1, Loginov Moscow Clinical Scientific Center of Moscow Department of Healthcare. http://orcid.org/0000-0001-7633-6852. E-mail: an.an.klimov@gmail.com

Anna A. Malakhova - ICU-physician of the ICU Department №1, Loginov Moscow Clinical Scientific Center of Moscow Department of Healthcare. http://orcid.org/0000-0002-2918-5091. E-mail: an.an.malahova@yandex.ru

Yakov A. Osokin - Resident of the ICU Department №1, Loginov Moscow Clinical Scientific Center of Moscow Department of Healthcare. http://orcid.org/0000-0003-4013-4970. E-mail: jamnstuff@gmail.com

For correspondence *: Valeriy V. Subbotin - Anesthesiology and ICU Department, Loginov Moscow Clinical Scientific Center, Department of Health of Moscow, 86, Shosse Entuziastov, Moscow, 111123, Russian Federation. Phone: +7-916-612-25-04. E-mail: v.subbotin@mknc.ru

Статья поступила в редакцию журнала 6.05.2019.

Received 6 May 2019.
Принята к публикации 28.05.2019. Accepted for publication 28 May 2019. 